\title{
On a multidimensional version of the Hilbert-type inequality in the whole plane
}

Predrag Vuković

\section{"Correspondence:}

predrag.vukovic@ufzg.hr

Faculty of Teacher Education,

University of Zagreb, Savska

cesta 77, Zagreb, 10000, Croatia

\begin{abstract}
The main objective of this paper is a study of some new multidimensional Hilbert-type inequalities with a general homogeneous kernel in the whole plane. We derive a pair of equivalent inequalities, and we also establish the conditions under which the constant factors included in the obtained inequalities are the best possible. Some applications in particular settings are also considered.

MSC: Primary 26D15

Keywords: Hilbert-type inequality; homogeneous kernel; the best possible constant; conjugate exponents
\end{abstract}

\section{Introduction}

Hilbert's inequality is one of the most significant weighted inequalities in mathematical analysis and its applications. Through the years, Hilbert-type inequalities were discussed by numerous authors, who either reproved them using various techniques, or applied and generalized them in many different ways. For more details as regards Hilbert's inequality the reader is referred to [1] or [2].

Although classical, Hilbert's inequality and its generalizations and modifications are still of a great interest. Xin and Yang in [3] proved Hilbert-type inequalities with the homogeneous kernel of degree -2 .

If $p>1,1 / p+1 / q=1,|\lambda|<1,0<\alpha_{1}<\alpha_{2}<\pi, f, g \geq 0$, satisfying $0<\int_{-\infty}^{\infty}|x|^{-p \lambda-1} f^{p}(x) d x<$ $\infty$ and $0<\int_{-\infty}^{\infty}|y|^{q \lambda-1} g^{q}(y) d y<\infty$, then we have

$$
\begin{aligned}
& \int_{-\infty}^{\infty} \int_{-\infty}^{\infty} \min _{i \in\{1,2\}}\left\{\frac{1}{x^{2}+2 x y \cos \alpha_{i}+y^{2}}\right\} f(x) g(y) d x d y \\
& <k(\lambda)\left(\int_{-\infty}^{\infty}|x|^{-p \lambda-1} f^{p}(x) d x\right)^{1 / p}\left(\int_{-\infty}^{\infty}|y|^{q \lambda-1} g^{q}(y) d y\right)^{1 / q}, \\
& \int_{-\infty}^{\infty}|y|^{p(1-\lambda)-1}\left(\int_{-\infty}^{\infty} \min _{i \in\{1,2\}}\left\{\frac{1}{x^{2}+2 x y \cos \alpha_{i}+y^{2}}\right\} f(x) d x\right)^{p} d y \\
& \quad<k^{p}(\lambda) \int_{-\infty}^{\infty}|x|^{-p \lambda-1} f^{p}(x) d x,
\end{aligned}
$$

where the constant factors

$$
k(\lambda)=\frac{\pi}{\sin \lambda \pi}\left[\frac{\sin \lambda \alpha_{1}}{\sin \alpha_{1}}+\frac{\sin \lambda\left(\pi-\alpha_{2}\right)}{\sin \alpha_{2}}\right] \quad(0<|\lambda|<1)
$$

and $k^{p}(\lambda)$ are the best possible. Inequalities (1.1) and (1.2) are equivalent.

\section{Springer}

๑2014 Vuković; licensee Springer. This is an Open Access article distributed under the terms of the Creative Commons Attribution License (http://creativecommons.org/licenses/by/2.0), which permits unrestricted use, distribution, and reproduction in any medium, provided the original work is properly cited. 
Our main objective is to emphasize the previous result. Our generalization will include a multidimensional version of the Hilbert-type inequality in the whole plane.

Some of the recent results concerning Hilbert's inequality include extension to multidimensional case, equipped with conjugate exponents $p_{i}$, that is, $\sum_{i=1}^{n} 1 / p_{i}=1, p_{i}>1, n \geq 2$ (see [4-6]). Here we refer to [7], which provides a unified treatment of the multidimensional Hilbert-type inequality in the setting with conjugate exponents. Suppose $\left(\Omega_{i}, \Sigma_{i}, \mu_{i}\right)$ are $\sigma$-finite measure spaces and $K: \prod_{i=1}^{n} \Omega_{i} \rightarrow \mathbb{R}, \phi_{i j}: \Omega_{j} \rightarrow \mathbb{R}, f_{i}: \Omega_{i} \rightarrow \mathbb{R}, i, j=1,2, \ldots, n$, are non-negative measurable functions. If $\prod_{i, j=1}^{n} \phi_{i j}\left(x_{j}\right)=1$, then the following inequalities hold and are equivalent:

$$
\int_{\mathbf{\Omega}} K(\mathbf{x}) \prod_{i=1}^{n} f_{i}\left(x_{i}\right) d \mu(\mathbf{x}) \leq \prod_{i=1}^{n}\left\|\phi_{i i} \omega_{i} f_{i}\right\|_{p_{i}}
$$

and

$$
\begin{aligned}
& {\left[\int_{\Omega_{n}} h\left(x_{n}\right)\left(\int_{\hat{\mathbf{\Omega}}^{n}} K(\mathbf{x}) \prod_{i=1}^{n-1} f_{i}\left(x_{i}\right) d \hat{\mu}^{n}(\mathbf{x})\right)^{q} d \mu\left(x_{n}\right)\right]^{1 / q}} \\
& \quad \leq \prod_{i=1}^{n-1}\left\|\phi_{i i} \omega_{i} f_{i}\right\|_{p_{i}},
\end{aligned}
$$

where

$$
\omega_{i}\left(x_{i}\right)=\left[\int_{\hat{\mathbf{\Omega}}^{i}} K(\mathbf{x}) \prod_{j=1, j \neq i}^{n} \phi_{i j}^{p_{i}}\left(x_{j}\right) d \hat{\mu}^{i}(\mathbf{x})\right]^{1 / p_{i}}
$$

and

$$
\begin{array}{ll}
1 / q=\sum_{i=1}^{n-1}\left(1 / p_{i}\right), & \boldsymbol{\Omega}=\prod_{i=1}^{n} \Omega_{i}, \quad \hat{\boldsymbol{\Omega}}^{i}=\prod_{j=1, j \neq i}^{n} \Omega_{j}, \quad \mathbf{x}=\left(x_{1}, x_{2}, \ldots, x_{n}\right), \\
h=\phi_{n n}^{-q} \omega_{n}^{1-q}, & d \mu(\mathbf{x})=\prod_{i=1}^{n} d \mu_{i}\left(x_{i}\right), \quad d \hat{\mu}^{i}(\mathbf{x})=\prod_{j=1, j \neq i}^{n} d \mu_{j}\left(x_{j}\right) .
\end{array}
$$

The abbreviations as in (1.6) will be used throughout the whole paper. Also note that $\|\cdot\|_{p_{i}}$ denotes the usual norm in $L^{p_{i}}\left(\Omega_{i}\right)$, that is,

$$
\left\|\phi_{i i} \omega_{i} f_{i}\right\|_{p_{i}}=\left[\int_{\Omega_{i}}\left(\phi_{i i} \omega_{i} f_{i}\right)^{p_{i}}\left(x_{i}\right) d \mu_{i}\left(x_{i}\right)\right]^{1 / p_{i}}, \quad i=1,2, \ldots, n .
$$

Our results will be based on the mentioned results of Yang et al. In what follows, without further explanation, we assume that all integrals exist on the respective domains of their definitions.

\section{Main results}

In this section we develop an unified treatment of the Hilbert and Hardy-Hilbert-type inequalities with general homogeneous kernel. Further, regarding the notations from the 
previous section, we assume that $\Omega_{i}=\mathbb{R}_{+}$, equipped with the non-negative Lebesgue measures $d \mu_{i}\left(x_{i}\right)=d x_{i}, i=1,2, \ldots, n$. In addition, we have $\boldsymbol{\Omega}=\mathbb{R}_{+}^{n}$ and $d \mathbf{x}=d x_{1} d x_{2} \cdots d x_{n}$.

Recall that the function $K: \mathbb{R}_{+}^{n} \rightarrow \mathbb{R}$ is said to be homogeneous of degree $-s, s>0$, if $K(t \mathbf{x})=t^{-s} K(\mathbf{x})$ for all $t>0$. Furthermore, for $\mathbf{a}=\left(a_{1}, a_{2}, \ldots, a_{n}\right) \in \mathbb{R}^{n}$, we define

$$
k_{i}(\mathbf{a})=\int_{\mathbb{R}^{n-1}} K\left(\hat{\mathbf{u}}^{i}\right) \prod_{j=1, j \neq i}^{n} u_{j}^{a_{j}} \hat{d}^{i} \mathbf{u}, \quad i=1,2, \ldots, n,
$$

where $\hat{\mathbf{u}}^{i}=\left(u_{1}, \ldots, u_{i-1}, 1, u_{i+1}, \ldots, u_{n}\right), \hat{d}^{i} \mathbf{u}=d u_{1} \cdots d u_{i-1} d u_{i+1} \cdots d u_{n}$, and provided that the above integral converges. Note that the constant factor $k_{i}(\mathbf{a})$ does not depend on the component $a_{i}$. Thus, the component $a_{i}$ can be replaced with an arbitrary real number. This fact will sometimes be used in the sequel, for reasons of simpler notation. Further, by using the substitutions $t_{i}=-u_{i}, i \neq 1$, we obtain the following identity:

$$
\int_{\mathbb{R}^{n-1}} K\left(-u_{1}, \ldots,-u_{i-1}, 1,-u_{i+1}, \ldots,-u_{n}\right) \prod_{j=1, j \neq i}^{n}\left|u_{j}\right|^{a_{j}} \hat{d}^{i} \mathbf{u}=k_{i}(\mathbf{a})
$$

for $i=1,2, \ldots, n$, where we assume that the above integral converges.

Utilizing inequalities (1.3) and (1.4) we obtain the following theorem.

Theorem 2.1 Let $p_{i}>1, i=1,2, \ldots, n$, be conjugate exponents and let $A_{i j}, i, j=1,2, \ldots, n$, be the real parameters such that $\sum_{i=1}^{n} A_{i j}=0, j=1,2, \ldots, n$. If $K: \mathbb{R}^{n} \rightarrow \mathbb{R}$ is a nonnegative measurable homogeneous function of degree $-\lambda, \lambda=2 m, m \in \mathbb{N}$, and $f_{i}: \mathbb{R} \rightarrow \mathbb{R}$, $i=1,2, \ldots, n$, are non-negative measurable functions, then the following inequalities hold and are equivalent:

$$
\int_{\mathbb{R}^{n}} K(\mathbf{x}) \prod_{i=1}^{n} f_{i}\left(x_{i}\right) d \mathbf{x} \leq \prod_{i=1}^{n} k_{i}^{1 / p_{i}}\left(p_{i} \mathbf{A}_{i}\right) \prod_{i=1}^{n}\left\|\left|x_{i}\right|^{(n-1-\lambda) / p_{i}+\alpha_{i}} f_{i}\right\|_{p_{i}}
$$

and

$$
\begin{aligned}
& {\left[\int_{\mathbb{R}}\left|x_{n}\right|^{(1-q)(n-1-\lambda)-q \alpha_{n}}\left(\int_{\mathbb{R}^{n-1}} K(\mathbf{x}) \prod_{i=1}^{n-1} f_{i}\left(x_{i}\right) \hat{d}^{n} \mathbf{x}\right)^{q} d x_{n}\right]^{1 / q}} \\
& \quad \leq \prod_{i=1}^{n} k_{i}^{1 / p_{i}}\left(p_{i} \mathbf{A}_{i}\right) \prod_{i=1}^{n-1}\left\|\left|x_{i}\right|^{(n-1-\lambda) / p_{i}+\alpha_{i}} f_{i}\right\|_{p_{i}}
\end{aligned}
$$

where $1 / q=\sum_{i=1}^{n-1}\left(1 / p_{i}\right), \alpha_{i}=\sum_{j=1}^{n} A_{i j}, \mathbf{A}_{i}=\left(A_{i 1}, A_{i 2}, \ldots, A_{\text {in }}\right), i=1,2, \ldots, n$, and $k_{i}(\cdot), i=$ $1,2, \ldots, n$, is defined by $(2.1)$.

Proof Rewrite inequality (1.3) for the functions $\phi_{i j}\left(x_{j}\right)=\left|x_{j}\right|^{A_{i j}}, i, j=1,2, \ldots, n$. Clearly, the set of the above defined power functions satisfies the condition

$$
\prod_{i, j=1}^{n} \phi_{i j}\left(x_{j}\right)=\prod_{j=1}^{n} \prod_{i=1}^{n} x_{j}^{A_{i j}}=\prod_{j=1}^{n} x_{j} \sum_{i=1}^{n} A_{i j}=1,
$$

since $\sum_{i=1}^{n} A_{i j}=0$. 
Obviously, it is enough to calculate the functions $\omega_{i}\left(x_{i}\right), i=1,2, \ldots, n$. Without loss of generality, we will take into account the function $\omega_{1}\left(x_{1}\right)$.

Now, when we express the function $\omega_{1}\left(x_{1}\right)$ in terms of the integral formula (2.1), we will use the following identity:

$$
\begin{aligned}
\int_{-\infty}^{\infty}\left(\phi_{11} \omega_{1} f_{1}\right)^{p_{1}}\left(x_{1}\right) d x_{1}= & \int_{-\infty}^{0}\left|x_{1}\right|^{p_{1} A_{11}} \omega_{1}^{p_{1}}\left(x_{1}\right) f_{1}^{p_{1}}\left(x_{1}\right) d x_{1} \\
& +\int_{0}^{\infty} x_{1}^{p_{1} A_{11}} \omega_{1}^{p_{1}}\left(x_{1}\right) f_{1}^{p_{1}}\left(x_{1}\right) d x_{1} .
\end{aligned}
$$

In the case when $x_{1} \leq 0, \lambda=2 m, m \in \mathbb{N}$, it follows that $x_{1}^{-\lambda}=\left|x_{1}\right|^{-\lambda}$. By using the substitutions $x_{i}=\left|x_{1}\right| u_{i}, i \neq 1$, the identity (2.2) and definition (2.1), we obtain the expression

$$
\begin{aligned}
\omega_{1}^{p_{1}}\left(x_{1}\right) & =\int_{\mathbb{R}^{n-1}} K(\mathbf{x}) \prod_{j=2}^{n}\left|x_{j}\right|^{p_{1} A_{1 j}} \hat{d}^{1} \mathbf{x} \\
& =\left|x_{1}\right|^{n-1-\lambda+p_{1}\left(\alpha_{1}-A_{11}\right)} \int_{\mathbb{R}^{n-1}} K\left(\hat{\mathbf{u}}^{1}\right) \prod_{j=2}^{n}\left|u_{j}\right|^{p_{1} A_{1 j}} \hat{d}^{1} \mathbf{u} \\
& =\left|x_{1}\right|^{n-1-\lambda+p_{1}\left(\alpha_{1}-A_{11}\right)} k_{1}\left(p_{1} \mathbf{A}_{1}\right) .
\end{aligned}
$$

Now, from (2.5) and (2.6) we get

$$
\left\|\phi_{11} \omega_{1} f_{1}\right\|_{p_{1}}=k_{1}\left(p_{1} \mathbf{A}_{1}\right) \int_{-\infty}^{\infty}\left|x_{1}\right|^{n-1-\lambda+p_{1} \alpha_{1}} f_{1}^{p_{1}}\left(x_{1}\right) d x_{1} .
$$

Similarly to the first part of the proof we obtain the identity

$$
\left\|\phi_{i i} \omega_{i} f_{i}\right\|_{p_{i}}=k_{i}\left(p_{i} \mathbf{A}_{i}\right) \int_{-\infty}^{\infty}\left|x_{i}\right|^{n-1-\lambda+p_{i} \alpha_{i}} f_{i}^{p_{i}}\left(x_{i}\right) d x_{i}, \quad i=1, \ldots, n .
$$

Finally, inequality (1.3) yields inequality (2.3). In the same way inequality (2.4) follows directly from (1.4).

The main idea in obtaining the best possible constant factor in inequalities (2.3) and (2.4) is a reduction of the constant in the form without exponents, by an appropriate choice of the parameters $\mathbf{A}_{i}, i=1, \ldots, n$. For that reason, we assume

$$
k_{1}\left(p_{1} \mathbf{A}_{1}\right)=k_{2}\left(p_{2} \mathbf{A}_{2}\right)=\cdots=k_{n}\left(p_{n} \mathbf{A}_{n}\right) .
$$

If we use the change of variables $u_{1}=1 / t_{2}, u_{3}=t_{3} / t_{2}, u_{4}=t_{4} / t_{2}, \ldots, u_{n}=t_{n} / t_{2}$, which provides the Jacobian of the transformation

$$
\left|\frac{\partial\left(u_{1}, u_{3}, \ldots, u_{n}\right)}{\partial\left(t_{2}, t_{3}, \ldots, t_{n}\right)}\right|=t_{2}^{-n}
$$

we have

$$
\begin{aligned}
k_{2}\left(p_{2} \mathbf{A}_{2}\right) & =\int_{\mathbb{R}_{+}^{n-1}} K\left(\hat{\mathbf{t}}^{1}\right) t_{2}^{\lambda-n-p_{2}\left(\alpha_{2}-A_{22}\right)} \prod_{j=3}^{n} t_{j}^{p_{2} A_{2 j}} \hat{d}^{1} \mathbf{t} \\
& =k_{1}\left(p_{1} A_{11}, \lambda-n-p_{2}\left(\alpha_{2}-A_{22}\right), p_{2} A_{23}, \ldots, p_{2} A_{2 n}\right) .
\end{aligned}
$$


According to (2.7), we have $p_{1} A_{12}=\lambda-n-p_{2}\left(\alpha_{2}-A_{22}\right), p_{1} A_{13}=p_{2} A_{23}, \ldots, p_{1} A_{1 n}=p_{2} A_{2 n}$. In a similar manner we express $k_{i}\left(p_{i} \mathbf{A}_{i}\right), i=3, \ldots, n$, in terms of $k_{1}(\cdot)$. To obtain a case of the best inequality it is natural to impose the following conditions on the parameters $A_{i j}$ :

$$
p_{j} A_{j i}=\lambda-n-p_{i}\left(\alpha_{i}-A_{i i}\right), \quad j \neq i, i, j \in\{1,2, \ldots, n\} .
$$

In that case the constant factor from Theorem 2.1 is simplified to the following form:

$$
L^{*}=k_{1}(\widetilde{\mathbf{A}}),
$$

where $\widetilde{\mathbf{A}}=\left(\widetilde{A}_{1}, \widetilde{A}_{2}, \ldots, \widetilde{A}_{n}\right)$ and

$$
\widetilde{A}_{i}=p_{1} A_{1 i} \quad \text { for } i \neq 1 \text { and } \widetilde{A}_{1}=p_{n} A_{n 1} \text {. }
$$

Further, by using (2.8) and (2.9), inequalities (2.3) and (2.4) with the parameters $A_{i j}$, satisfying (2.8), become

$$
\int_{\mathbb{R}^{n}} K(\mathbf{x}) \prod_{i=1}^{n} f_{i}\left(x_{i}\right) d \mathbf{x} \leq L^{*} \prod_{i=1}^{n}\left\|\left|x_{i}\right|^{-\tilde{A}_{i}-1 / p_{i}} f_{i}\right\|_{p_{i}}
$$

and

$$
\begin{aligned}
& {\left[\int_{\mathbb{R}}\left|x_{n}\right|^{(1-q)\left(-1-p_{n} \widetilde{A}_{n}\right)}\left(\int_{\mathbb{R}^{n-1}} K(\mathbf{x}) \prod_{i=1}^{n-1} f_{i}\left(x_{i}\right) \hat{d}^{n} \mathbf{x}\right)^{q} d x_{n}\right]^{1 / q}} \\
& \quad \leq L^{*} \prod_{i=1}^{n-1}\left\|\left|x_{i}\right|^{-\widetilde{A}_{i}-1 / p_{i}} f_{i}\right\|_{p_{i}} .
\end{aligned}
$$

To prove the main result we need the next lemma.

Lemma 2.2 Let $K: \mathbb{R}_{+}^{n} \rightarrow \mathbb{R}$ be a non-negative measurable homogeneous function of degree $-\lambda, \lambda=2 m, m \in \mathbb{N}$, such that for every $i=2,3, \ldots, n$,

$$
K\left(1, t_{2}, \ldots, t_{i}, \ldots, t_{n}\right) \leq C K\left(1, t_{2}, \ldots, 0, \ldots, t_{n}\right), \quad-1 \leq t_{i} \leq 1
$$

where $C$ is a positive constant. Let the parameters $\widetilde{A}_{i}, i=1, \ldots, n$, be defined by (2.10) and $0<\varepsilon<\min _{1 \leq i \leq n}\left\{p_{i}+p_{i} \widetilde{A}_{i}\right\}$. Then we have

$$
\begin{aligned}
& \int_{\left|x_{1}\right| \geq 1}\left|x_{1}\right|^{\widetilde{A}_{1}-\varepsilon / p_{1}}\left(\sum_{i=2}^{n} \int_{D_{i}^{-}} K(\mathbf{x}) \prod_{j=2}^{n}\left|x_{j}\right|^{\widetilde{A}_{j}-\varepsilon / p_{j}} \hat{d}^{1} \mathbf{x}\right) d x_{1} \\
& +\int_{\left|x_{1}\right| \geq 1}\left|x_{1}\right|^{\widetilde{A}_{1}-\varepsilon / p_{1}}\left(\sum_{i=2}^{n} \int_{D_{i}^{+}} K(\mathbf{x}) \prod_{j=2}^{n}\left|x_{j}\right|^{\widetilde{A}_{j}-\varepsilon / p_{j}} \hat{d}^{1} \mathbf{x}\right) d x_{1} \leq O(1),
\end{aligned}
$$

where

$$
\begin{aligned}
& D_{i}^{-}=\left\{\left(x_{2}, \ldots, x_{n}\right):-1 \leq x_{i}<0, x_{j} \in \mathbb{R}, j \neq i\right\}, \\
& D_{i}^{+}=\left\{\left(x_{2}, \ldots, x_{n}\right): 0<x_{i} \leq 1, x_{j} \in \mathbb{R}, j \neq i\right\}, \quad i=2, \ldots, n .
\end{aligned}
$$


Proof First, we define the integral $I_{j}\left(x_{1}\right), j=2, \ldots, n$, by the formulas

$$
I_{j}\left(x_{1}\right)=\int_{D_{j}^{-}} K(\mathbf{x}) \prod_{i=2}^{n}\left|x_{i}\right|^{\tilde{A}_{i}-\varepsilon / p_{i}} \hat{d}^{1} \mathbf{x}
$$

where $D_{j}^{-}$is defined by (2.15). Without loss of generality we only estimate the integral $I_{2}\left(x_{1}\right)$. By using homogeneity of the function $K$, the substitutions $t_{i}=x_{i} /\left|x_{1}\right|, i \neq 1$, and the condition (2.13), we obtain

$$
\begin{aligned}
I_{2}\left(x_{1}\right)= & \left|x_{1}\right|^{\sum_{i=2}^{n}\left(\widetilde{A}_{i}-\varepsilon / p_{i}\right)+n-1-\lambda} \int_{\mathbb{R}^{n-2}} \int_{0}^{1 /\left|x_{1}\right|} K\left(\hat{\mathbf{t}}^{1}\right) \prod_{i=2}^{n}\left|t_{i}\right|^{\widetilde{A}_{i}-\varepsilon / p_{i}} \hat{d}^{1} \mathbf{t} \\
\leq & C\left|x_{1}\right|^{\sum_{i=2}^{n}\left(\widetilde{A}_{i}-\varepsilon / p_{i}\right)+n-1-\lambda} \int_{\mathbb{R}^{n-2}} K\left(1,0, t_{3}, \ldots, t_{n}\right) \prod_{i=3}^{n}\left|t_{i}\right|^{\widetilde{A}_{i}-\varepsilon / p_{i}} d t_{3} \cdots d t_{n} \\
& \times \int_{0}^{1 /\left|x_{1}\right|} t_{2}^{\widetilde{A}_{2}-\varepsilon / p_{2}} d t_{2} \\
= & C\left(1-\varepsilon / p_{2}+\widetilde{A}_{2}\right)^{-1}\left|x_{1}\right|^{\varepsilon / p_{2}-\widetilde{A}_{2}-1} k_{1}\left(\widetilde{A}_{1}-\varepsilon / p_{1}, \widetilde{A}_{3}-\varepsilon / p_{3}, \ldots, \widetilde{A}_{n}-\varepsilon / p_{n}\right) \\
& \times\left|x_{1}\right|^{\sum_{i=2}^{n}\left(\widetilde{A}_{i}-\varepsilon / p_{i}\right)+n-1-\lambda} .
\end{aligned}
$$

Hence, we have

$$
I_{j}\left(x_{1}\right) \leq\left|x_{1}\right|^{\varepsilon / p_{j}-\widetilde{A}_{j}-1}\left|x_{1}\right|^{\sum_{i=2}^{n}\left(\widetilde{A}_{i}-\varepsilon / p_{i}\right)+n-1-\lambda} O_{j}(1)
$$

for $\varepsilon \rightarrow 0^{+}, j \in\{2, \ldots, n\}$, and consequently

$$
\int_{\left|x_{1}\right| \geq 1}\left|x_{1}\right|^{\widetilde{A}_{1}-\varepsilon / p_{1}} \sum_{j=2}^{n} I_{j}\left(x_{1}\right) d x_{1} \leq O(1) .
$$

In a similar manner we obtain inequality (2.16) when in the definition of the integral $I_{j}\left(x_{1}\right)$ the space is $D_{j}^{-}$replaced by $D_{j}^{+}$. Finally, from (2.16) we get (2.15).

Now, we are ready to state and prove the main result, concerning the best possible constant factor in inequalities (2.11) and (2.12).

Theorem 2.3 Let the kernel $K$ and the parameters $A_{i j}, i, j=1, \ldots, n$, be defined as in Theorem 2.1. If the kernel $K$ and the parameters $A_{i j}$ satisfy the conditions (2.13) and (2.8), respectively, then the constant $L^{*}$ is the best possible in inequalities (2.11) and (2.12).

Proof Let us suppose that the constant factor $L^{*}$ given by (2.9) is not the best possible in inequality (2.11). Then there exists a positive constant $L_{1}<L^{*}$, such that (2.11) is still valid when we replace $L^{*}$ by $L_{1}$.

We define the real functions $\tilde{f}_{i, \varepsilon}: \mathbb{R}^{n} \mapsto \mathbb{R}$ by the formulas

$$
\tilde{f}_{i, \varepsilon}\left(x_{i}\right)=\left\{\begin{array}{ll}
0, & \left|x_{i}\right|<1, \\
\left|x_{i}\right|^{\widetilde{A}_{i}-\varepsilon / p_{i}}, & \left|x_{i}\right| \geq 1,
\end{array} \quad i=1, \ldots, n\right.
$$


where $0<\varepsilon<\min _{1 \leq i \leq n}\left\{p_{i}+p_{i} \widetilde{A}_{i}\right\}$. Now, we shall put these functions in inequality (2.11). Then the right-hand side of inequality (2.11) becomes

$$
L_{1} \prod_{i=1}^{n}\left[\int_{\left|x_{i}\right| \geq 1}\left|x_{i}\right|^{-1-\varepsilon} d x_{i}\right]^{1 / p_{i}}=2 L_{1} \int_{1}^{\infty} t^{-1-\varepsilon} d t=\frac{2 L_{1}}{\varepsilon} .
$$

Further, let $J$ denotes the left-hand side of inequality (2.11), for the above choice of the functions $\tilde{f}_{i, \varepsilon}$. Now, it is easy to see that the following inequality holds:

$$
\begin{aligned}
J \geq & \int_{\left|x_{1}\right| \geq 1}\left|x_{1}\right|^{\widetilde{A}_{1}-\varepsilon / p_{1}}\left(\int_{\mathbb{R}^{n-1}} K(\mathbf{x}) \prod_{j=2}^{n}\left|x_{j}\right|^{\widetilde{A}_{j}-\varepsilon / p_{j}} \hat{d}^{1} \mathbf{x}\right) d x_{1} \\
& -\int_{\left|x_{1}\right| \geq 1}\left|x_{1}\right|^{\widetilde{A}_{1}-\varepsilon / p_{1}}\left(\sum_{i=2}^{n} \int_{D_{i}^{-}} K(\mathbf{x}) \prod_{j=2}^{n}\left|x_{j}\right|^{\widetilde{A}_{j}-\varepsilon / p_{j}} \hat{d}^{1} \mathbf{x}\right) d x_{1} \\
& -\int_{\left|x_{1}\right| \geq 1}\left|x_{1}\right|^{\widetilde{A}_{1}-\varepsilon / p_{1}}\left(\sum_{i=2}^{n} \int_{D_{i}^{+}} K(\mathbf{x}) \prod_{j=2}^{n}\left|x_{j}\right|^{\widetilde{A}_{j}-\varepsilon / p_{j}} \hat{d}^{1} \mathbf{x}\right) d x_{1},
\end{aligned}
$$

where $D_{i}^{-}, D_{i}^{+}, i=2, \ldots, n$, are defined by (2.15). By using the substitutions $u_{i}=x_{i} /\left|x_{1}\right|, i \neq 1$, and Lemma 2.2 we obtain

$$
\begin{aligned}
J & \geq \int_{\left|x_{1}\right| \geq 1}\left|x_{1}\right|^{\widetilde{A}_{1}-\varepsilon / p_{1}}\left(\int_{\mathbb{R}^{n-1}} K\left(\hat{\mathbf{u}}^{1}\right) \prod_{j=2}^{n}\left|u_{j}\right|^{\widetilde{A}_{j-\varepsilon} / p_{j}} \hat{d}^{1} \mathbf{u}\right) d x_{1}-O(1) \\
& =\frac{2}{\varepsilon} k_{1}(\widetilde{\mathbf{A}}-\varepsilon \mathbf{1} / \mathbf{p})-O(1),
\end{aligned}
$$

where $\widetilde{\mathbf{A}}-\varepsilon \mathbf{1} / \mathbf{p}=\left(\widetilde{A}_{1}-\varepsilon / p_{1}, \widetilde{A}_{2}-\varepsilon / p_{2}, \ldots, \widetilde{A}_{n}-\varepsilon / p_{n}\right)$. From (2.11), (2.17), and (2.18) we get

$$
k_{1}(\widetilde{\mathbf{A}}-\varepsilon \mathbf{1} / \mathbf{p})-o(1) \leq L_{1} .
$$

Now, by letting $\varepsilon \rightarrow 0^{+}$we obtain $L^{*} \leq L_{1}$ which contradicts with the assumption $L_{1}<L^{*}$. Thus, the constant $L^{*}$ is the best possible.

Finally, the equivalence of inequalities (2.11) and (2.12) means that the constant $L^{*}$ is also the best possible in inequality (2.12). That completes the proof.

It is easy to see that the parameters $A_{i j}, i, j=1, \ldots, n$, defined by

$$
A_{i j}=\frac{\lambda-p_{j}}{p_{i} p_{j}}, \quad i \neq j \quad \text { and } \quad A_{i i}=\frac{\left(\lambda-p_{i}\right)\left(1-p_{i}\right)}{p_{i}^{2}}
$$

satisfy the conditions (2.8).

Setting $n=3$ and the parameters $\widetilde{A}_{1}=\left(\lambda-p_{1}\right) / p_{1}, \widetilde{A}_{2}=\left(\lambda-p_{2}\right) / p_{2}, \widetilde{A}_{3}=\left(\lambda-p_{3}\right) / p_{3}$ in inequalities (2.11) and (2.12) we obtain the following result.

Corollary 2.4 Let $p_{1}, p_{2}, p_{3}$ be conjugate parameters such that $p_{i}>1, i=1,2,3$, and let $1 / q=1 / p_{1}+1 / p_{2}$. Let $K: \mathbb{R}^{3} \rightarrow \mathbb{R}$ be non-negative measurable homogeneous function of 
degree $-\lambda, \lambda=2 m, m \in \mathbb{N}$, satisfying condition (2.13). If $f_{i}: \mathbb{R} \rightarrow \mathbb{R}, i=1,2,3$, are nonnegative measurable functions, then the following inequalities hold and are equivalent:

$$
\int_{\mathbb{R}^{3}} K\left(x_{1}, x_{2}, x_{3}\right) \prod_{i=1}^{3} f_{i}\left(x_{i}\right) d x_{1} d x_{2} d x_{3} \leq M \prod_{i=1}^{3}\left\|\left|x_{i}\right|^{\left(p_{i}-\lambda-1\right) / p_{i}} f_{i}\right\|_{p_{i}}
$$

and

$$
\begin{aligned}
& {\left[\int_{\mathbb{R}}\left|x_{3}\right|^{\lambda /\left(p_{3}-1\right)-1}\left(\int_{\mathbb{R}^{2}} K\left(x_{1}, x_{2}, x_{3}\right) f_{1}\left(x_{1}\right) f_{2}\left(x_{2}\right) d x_{1} d x_{2}\right)^{q} d x_{3}\right]^{1 / q}} \\
& \quad \leq M \prod_{i=1}^{2}\left\|\left|x_{i}\right|^{\left(p_{i}-\lambda-1\right) / p_{i}} f_{i}\right\|_{p_{i}},
\end{aligned}
$$

where the constant

$$
M=k\left(\frac{\lambda-p_{1}}{p_{1}}, \frac{\lambda-p_{2}}{p_{2}}, \frac{\lambda-p_{3}}{p_{3}}\right)
$$

is the best possible in inequalities (2.20) and (2.21).

Remark 2.5 Note that $K\left(x_{1}, x_{2}, x_{3}\right)=\left(\left|x_{1}\right|+\left|x_{2}\right|+\left|x_{3}\right|\right)^{-\lambda}, \lambda=2 m, m \in \mathbb{N}$, is a homogeneous function of degree $-\lambda$. In this case using Corollary 2.4 and the formula (see [8])

$$
\int_{(0, \infty)^{n-1}} \frac{\prod_{i=1}^{n-1} u_{i}^{r_{i}-1}}{\left(1+\sum_{i=1}^{n-1} u_{i}\right)^{\sum_{i=1}^{n} r_{i}}} d u_{1} \cdots d u_{n-1}=\frac{\prod_{i=1}^{n} \Gamma\left(r_{i}\right)}{\Gamma\left(\sum_{i=1}^{n} r_{i}\right)}, \quad r_{i}>0, i=1, \ldots, n,
$$

we obtain the best possible constant $M=4 / \Gamma(\lambda) \prod_{i=1}^{3} \Gamma\left(\lambda / p_{i}\right)$.

Remark 2.6 The kernel $K_{1}(x, y)=\min _{i \in\{1,2\}}\left\{1 /\left(x^{2}+2 x y \cos \alpha_{i}+y^{2}\right)\right\}, 0<\alpha_{1}<\alpha_{2}<\pi$, is homogeneous function of degree -2 . By putting the kernel $K_{1}(x, y)$ and the parameters $A_{1}=-\lambda / q$ and $A_{2}=\lambda / p$ in inequalities (2.11) and (2.12) we obtain the result of Xin et al. (see also [3]).

\section{Competing interests}

The author declares that they have no competing interests.

\section{Acknowledgements}

This research was supported under Research Grants 202339 and 058-1170889-1050.

Received: 7 February 2014 Accepted: 28 June 2014 Published: 23 Jul 2014

\section{References}

1. Hardy, GH, Littlewood, JE, Pólya, G: Inequalities, 2nd edn. Cambridge University Press, Cambridge (1967)

2. Mitrinović, DS, Pečarić, J, Fink, AM: Classical and New Inequalities in Analysis. Kluwer Academic, Dordrecht (1993)

3. Xin, D, Yang, B: A Hilbert-type integral inequality in the whole plane with the homogeneous kernel of degree -2 . J. Inequal. Appl. 2011, Article ID 401428 (2011)

4. Huang, Q, Yang, B: On a multiple Hilbert-type integral operator and applications. J. Inequal. Appl. 2009, Article ID 192197 (2009)

5. Vuković, P: Note on Hilbert-type inequalities. Turk. J. Math. 35, 1-10 (2011)

6. Krnić, M, Pečarić, J, Perić, I, Vuković, P: Recent Advances in Hilbert-Type Inequalities. Element, Zagreb (2012)

7. Yang, B, Brnetić, I, Krnić, M, Pečarić, J: Generalization of Hilbert and Hardy-Hilbert integral inequalities. Math. Inequal. Appl. 8(2), 259-272 (2005)

8. Rassias, TM, Yang, B: On the way of weight coefficient and research for the Hilbert-type inequalities. Math. Inequal. Appl. 6(2), 625-658 (2003) 
10.1186/1029-242X-2014-272

Cite this article as: Vuković: On a multidimensional version of the Hilbert-type inequality in the whole plane. Journal of Inequalities and Applications 2014, 2014:272

Submit your manuscript to a SpringerOpen ${ }^{\circ}$ journal and benefit from:

- Convenient online submission

- Rigorous peer review

- Immediate publication on acceptance

Open access: articles freely available online

- High visibility within the field

- Retaining the copyright to your article

Submit your next manuscript at $\boldsymbol{s p r i n g e r o p e n . c o m ~}$ 